\title{
Neural Measures Reveal Human Short-Term Working Memory -Varying Both Load and Cue Size
}

\author{
Ningning Zhang ${ }^{1, \text { a }}$, Hong Wang ${ }^{1, \text { b }}$ \\ ${ }^{1}$ School of mechanical engineering and automation Northeastern University, China \\ aznninging@yahoo.com.cn, bhongwang@mail.neu.edu.cn
}

Keywords: Short-term working memory, Load, Cue size, Time latency, EEG

\begin{abstract}
The objective of the study was to investigate neural processing during short-term visual working memory task under different memory load and cue size. Two or four items of the same sign but in different colors were presented on both sides of the screen, in which cues were applied as an indicator. Participants were cued to remember the colors of one side and then make a quickly response to the test sample. On the performance level, responses accuracy decreased with the increasing numbers of working memory loads. The response time increased with the increasing numbers of working memory load. The response time latency to the big cue was larger than that of the small cue. On the neural processing level, the high-load stimuli could arouse a more negative wave than the low-load stimuli.
\end{abstract}

\section{Introduction}

Working memory is considered as a system that temporarily generates and maintains a representation of a perceived stimulus in mind that can be accessed and manipulated in the context of performing cognitive tasks [1]. Working memory processes consist of three processing stages: (1) a stimulus is perceived and a representation of it is generated in mind (encoding), (2) this representation is maintained in mind (retention) for several seconds, (3) this representation is accessed for performing a cognitive task (retrieval). The most influential model of working memory probably was the tripartite model. According to the model, working memory consists of three components: the phonological loop, the visuo-spatial sketchpad and the episodic buffer [2]. The visuo-spatial working memory was discussed in present study.

Event-related potential (ERPs) is the brain electricity potentials by the average addition of the EEG data. Event-related potential (ERPs) with their high temporal resolution allow to investigate the time course of the different neuronal processing phases (encoding, retention, and retrieval) during a working memory task distinctly. In addition, the effects of varying working memory load on these neural processes can be evaluated [3].

There are a variety of tasks used in working memory paradigms, with delayed match-to-sample task and n-back tasks belonging to the most common ones. In delayed match- to-sample tasks, several items are presented simultaneously (encoding), next a retention period is included, which is followed by a probe stimulus (retrieval). In n-back task, items are presented consecutively and the task is to indicate whether the current stimulus matched the one presented $n$ trials before [4]. The delayed match-to-sample task was applied in present study.

Working memory is an attention-demanding system which is affected by load and which has a limited capacity [5]. The capacity of the working memory is a hot topic, which is supposed to have three to four items [6]. Higher cognitive activity was observed during remembering color conjunctions than colors [7]. This suggests that maintenance is not for free, it causes additional effort. The observed decrease of memory with increasing set size in the conjunction condition must be caused by different effect. However, not only the number of the objects but also sensory load limits visual working memory [8]. The effects of working memory load have been analyzed in several studies on event-related potential components focusing inter alia on P3 amplitudes. They provide converging evidence that P3 amplitudes decrease with increasing working memory load [9]. The capacity decreased when complexity of the objects increased [10]. The capacity was perfectly related to the search rate in visual search paradigm. 
The present study addressed this issue of cued working memory between different numbers of stimuli. The physical properties (such as orientation, location, and spatial frequency) and sensory interactions were balanced through different numbers of stimuli load conditions. This allows us to determine whether the load differences under different cue size could evoke neural processing differences.

\section{Methods}

Participants. Twenty healthy right-handed volunteers ranging in age from 18 to 30 years old, with normal or corrected-to-normal visual acuity participated in this study. All participants were unaware of the rationale of the experiment, filled out a consent form prior to the experiment.

Stimuli. There are four stimulations in the experiment, varying both load and cue size. Different loads means two items in a trial or four items in a trial. There are two different cue sizes: big cue and small cue. The items, composed of oriented lines, are in different colors but in same shape in a trial. That is to say there's only a color difference between figures in a trial, both two sides. There're seven colors in all: red, blue, green, violet, yellow, white and black. The arrangement of the four items is square, and the two items are developed from four items by subtracting two. Only one item changed color or none item changed color in a trial.

The stimulations are indicated by serious cues (See Fig.1). Firstly, participants will see an arrow above the fixation cross which is in the center of the screen. Attend to the side of the screen indicated by the arrow; participants were told to try to keep eyes in the center at the fixation cross. Next, participants may see a cue (a box) that will appear somewhere on the screen. Two or four items will appear in the cue (the box). Participants need to remember the colors of the items. During the retention interval, wait and remember the colors. When test array present, participants are supposed to give a decision if the color has changed. Participants should response as accurately as quickly as possible. After press a key, a new trial will begin immediately. And response must be within 2 seconds. If it takes too long, the program will move onto other trial.



Fig.1. Illustrations of the cue and the stimulation for a trial in the study

Software Eprime and SCAN 4.4.3 were used to present stimuli and to record and analyze EEG. The subjects wore the electrode cap with $6.8 \mathrm{k} \Omega$ electrode impedances. 28 channels of EEG and EOG were recorded from the scalp with the electrode cap. EEG was recorded continuously with amplifiers using a 64-channel $\mathrm{Ag} / \mathrm{AgCl}$ electrode net. Acquisition software was running on a power computer.

After recording, EEG data were obtained and were ready to be analyzed. Firstly, the behavioral data were immerged. Artifacts from eye movements were removed from individual trials using the procedure. The exquisite dithered waves were wiped off because it will affect the final results. Then EEG was segmented with a pre-stimulus baseline of subtractive $100 \mathrm{~ms}$ and $1000 \mathrm{~ms}$ pos-stimulus epoch. The artifacts were rejected, making sure the rejected numbers were less than twenty percent of the total numbers. ERP data were averaged according different rules. Then the data were filtered by a low pass filter. The frequency of normal brain waves is less than $30 \mathrm{~Hz}$, so a $30 \mathrm{~Hz}$ low pass filter was applied.

\section{Results}

Behavioral Results. For behavioral data analysis, average response time and accuracy were used for satistical analysis. Participants responded faster under the small cue than the big cue (see Fig.2). A significance effect was obtained for the response time under different cue sizes of stimuli $(\mathrm{F}=8.35$, 
$\mathrm{P}<.05$ ). Participants responded faster to the low-load (two items) stimuli relative to the high-load (four items) stimuli (see Fig.2). The accuracy for two items is higher (see Fig.3). A significance effect was obtained for the accuracy under different numbers of stimuli $(\mathrm{F}=56.37, \mathrm{P}<.001)$. There's no significant difference in accuracy between the big cue and small cue $(\mathrm{P}>.05)$.

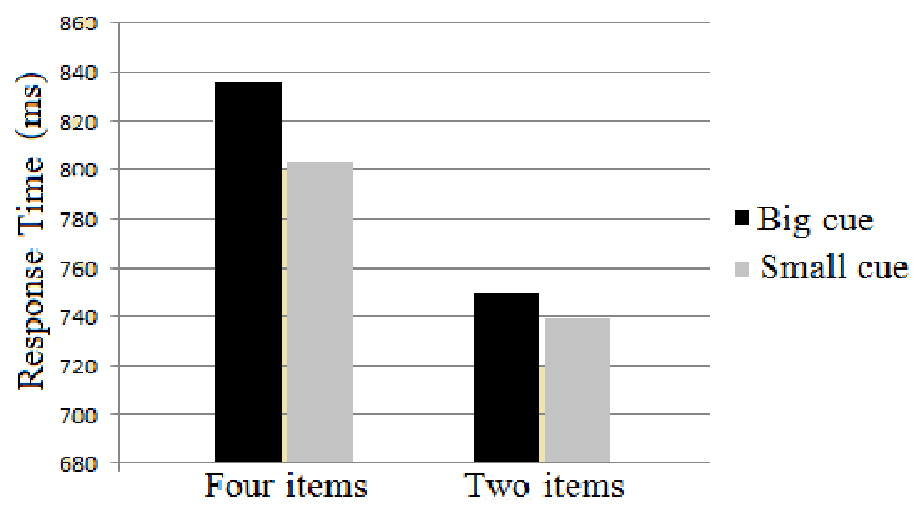

Fig.2. The average response time under different load and cue size

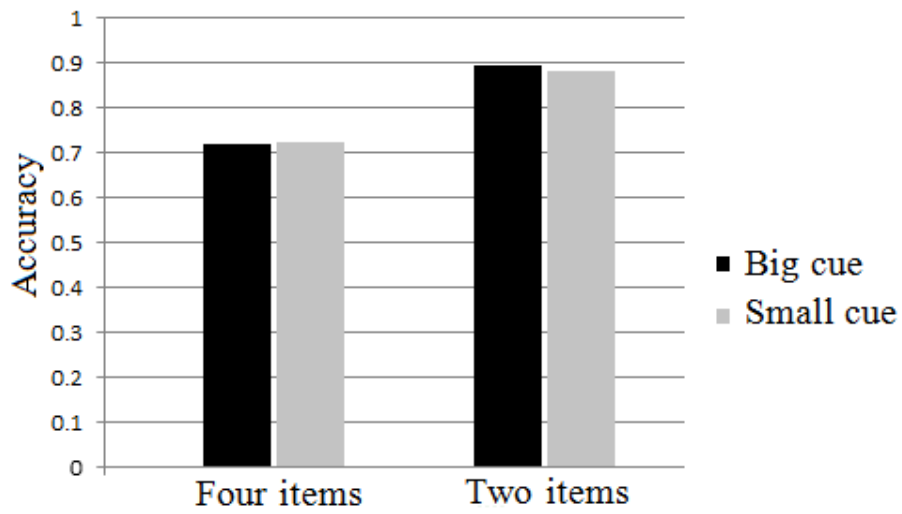

Fig.3. Average response accuracy under different load and cue size

ERP Results. ERP is getting from the average addition of the EEG data. The grand average ERP data illustrates participants' neural processing during encoding, retention and retrieval in the working memory task. The latency windows were: $\mathrm{P} 1=80-120 \mathrm{~ms}, \mathrm{~N} 1=120-160 \mathrm{~ms}, \mathrm{P} 2=160-220 \mathrm{~ms}$, $\mathrm{N} 2=220-300 \mathrm{~ms}, \mathrm{P} 3=300-450 \mathrm{~ms}$, early slow wave $(\mathrm{SW} 1)=550-700 \mathrm{~ms}$, late slow wave (SW2) $=700-850 \mathrm{~ms}$. For the ERP data analysis, the P3 and early slow waves reflected the retrieval process during the task. The prefrontal cortex is a part of brain which reflects working memory activities. The four items could arouse more negative waves in the P3 and early slow waves at the prefrontal cortex (electrodes F3, FZ, F4, see Fig.4). We still can not see the brain wave differences between different cue size.

Both the perceptive high-load and the increase of stimuli numbers could limit the capacity of working memory. This suggests that pay attention to large numbers of items is not for free, it causes additional effort compare to a small numbers of items.

\section{Discussion}

The behavioral data shows that there've significant differences between the different loads and cue sizes. Responses accuracy decreases with the increasing numbers of working memory loads. 


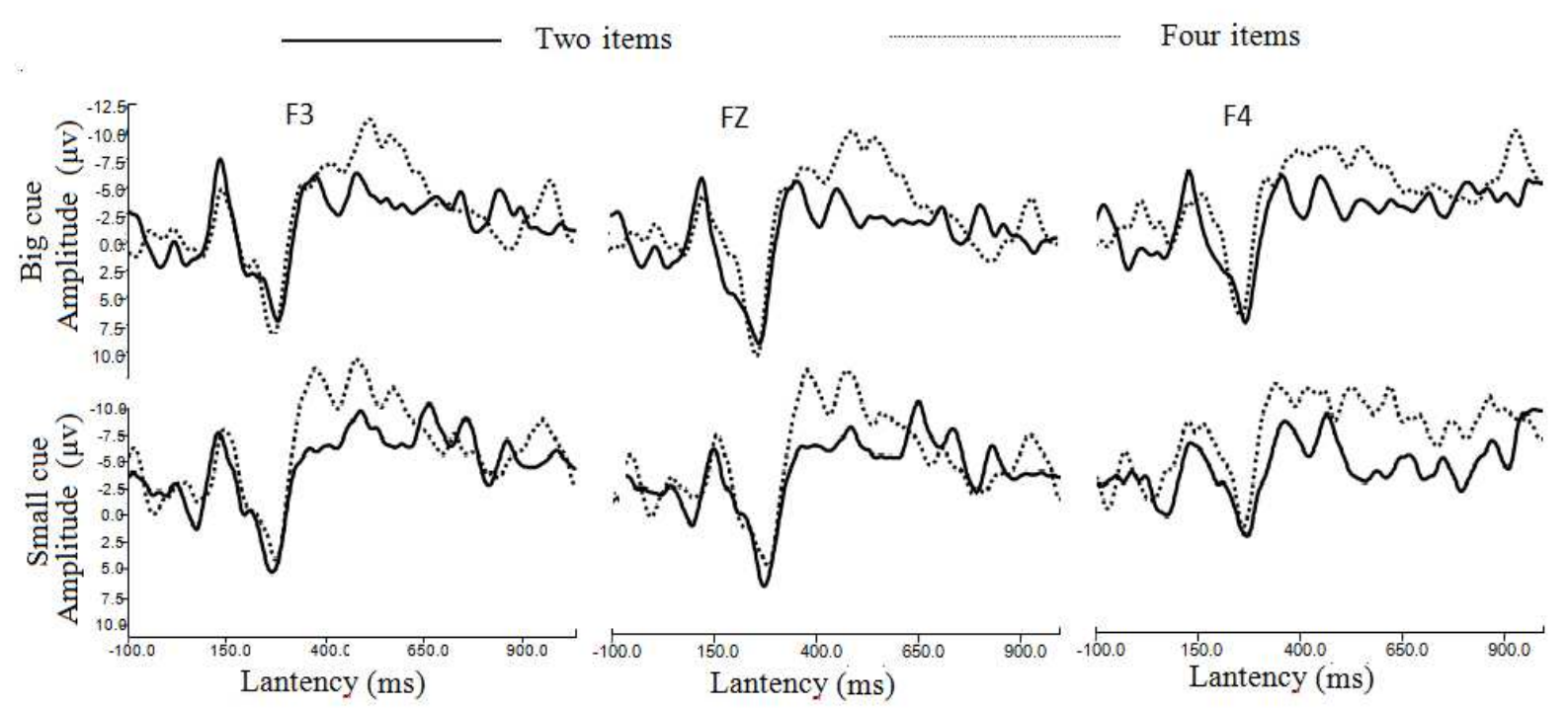

Fig.4. Illustrations of participants' grand average ERP data on electrodes F3, FZ, F4

Response time increases with the increasing numbers of working memory load. The response time latency to the big cue is larger than that of the small cue. The ERP data shows that the four items could evoke higher amplitude on prefrontal cortex. This suggests that paying attention to more items will cause a more negtive waves on the prefrontal cortex.

\section{Acknowledgement}

This work was supported by National Science Foundation of China (61071057), Fundamental Research Funds for the Central Universities of China (N100603003). We also would like to express our appreciation to the faculty of Arch Lab.

\section{References}

[1] A. Baddeley, Working memory, Science 255 (1992) 556-559.

[2] K.J. Gilhooly, R.H. Logie, Thinking in working memory, in: K.J. Gilhooly, R.H. Logie (Eds.), Working memory and thinking, Current issues in thinking and reasoning, Psychology Press Ltd., East Sussex, 1998, pp. 1-6.

[3] S. Fu, J. Fedota, G. M. Pamela, R. Parasuraman. Early interaction between perceptual load and involuntary attention: An event-related potential study, J. Neuroscience Letters 468 (2010) 68-71.

[4] S. Petra, W. Susanne, D. Martin S, ERP effects of methylphenidate and working memory load in healthy adults during a serial visual working memory task, J. Neuroscience Letters 482 (2010) 172-176

[5] D. Hubert, Zimmer, Visual and spatial working memory: From boxes to networks, J. Neuroscience and Biobehavioral Reviews 32(2008) 1373-1395.

[6] H. Shibuya, C. Bundesen, Visual selection from multielement displays: measuring and modeling effects effects of exposure duration, J. Exp. Psychol. Hum. Percept. Perform. 14(4), (1988) 591-600.

[7] S.P.Marshall, C.L. Davis, S.R. Knust, The index of cognitive activity: estimating cognitive effort from pupil dilation. San Diego State University and Eye Tracking Inc. San Diego. (2004)

[8] G.A. Alvarez, P. Cavanagh. The capacity of visual short term memory is set both by visual information load and by number of objects. J. Psychol. Sci. 15(2), (2004) 106-111.

[9] A. Kok, On the utility of P3 amplitude as a measure of processing capacity, J. Psychophysiology 38 (2001) 557-577.

[10] E.K. Vogel, M.G. Machizawa, Neural activity predicts individual differences in visual working memory capacity. J. Nature 428, (2004) 748-751. 